\title{
B-17-2020
}

\section{ANÁLISIS DE UNA BOMBA TRABAJANDO COMO TURBINA PARA LA RECUPERACIÓN DE ENERGÍA EN UNA RED DE RIEGO DE LA C.R. PICASSENT (SECTOR IV)}

\author{
Gómez Bonastre, A (1) (P), Palau Estevan C.V. (2), Balbastre Peralta, I. (3)
}

\begin{abstract}
${ }^{1}$ Estudiante de Máster Ingeniería Agronómica, Universitat Politècnica de València (UPV), Camino de Vera s/n, 46022 Valencia, algobo@etsiamn.upv.es

2 Docente e Investigadora, Dpto. DIRA, Universitat Politècnica de Valencia, Camino de Vera s/n, 46022 Valencia, virpaes@agf.upv.es

${ }^{3}$ Docente, Investigador y Responsable del Laboratorio Ingeniería Rural, Hidráulica y Riegos

(LIHR), Dpto. DIRA, Universitat Politècnica de Valencia, Camino de Vera s/n, 46022

Valencia, ibbalpe@agf.upv.es
\end{abstract}

\section{Resumen}

El objetivo del presente trabajo es el análisis energético de un sistema de riego en el Sector IV de la Comunidad de Regantes de Picassent para instalar una bomba trabajando como turbina (Pump As Turbine, PAT). El análisis de la eficiencia energética en redes de riego permite determinar y aprovechar la energía hidráulica disponible en el sistema de riego con el objetivo de obtener una instalación lo más autosuficiente posible. En este trabajo se estudiará a través de simulaciones en EPANET una red abastecida por gravedad desde la balsa de San Rafael (T. M. Picassent) a una cota de $165 \mathrm{~m}$ hasta una zona regable donde los hidrantes multiusuario reciben presiones excesivas a cotas en torno a $95 \mathrm{~m}$.

Para ello se decide instalar una PAT que permita recuperar el posible excedente de energía, estudiando cuatro escenarios diferentes de distribución del riego. La zona regable tiene diferentes cultivos leñosos en producción, con una dominancia de los cítricos, teniendo por otro lado aguacates y caquis. Estos cultivos cubren una superficie aproximada de 30 ha. Se realizará un diseño agronómico para estimar las necesidades de riego que tienen los cultivos durante el año y la posible organización del riego en función de los escenarios contemplados.

Finalmente, se propone un nuevo modelo de la red con las demandas de agua previstas y se estima la energía recuperable para cada escenario garantizando el correcto funcionamiento del sistema a lo largo de la campaña de riego. De esta forma, se concluirá sobre las condiciones idóneas para la implantación de la PAT en una red de riego.

\section{"Abstract"}

The aim of this project is the energetic analysis of an irrigation system belonging to the Water Users Association (WUA) of Picassent's municipality, located in the Sector IV, and assess the possibility of installing a Pump working as a Turbine (PAT). This type of analysis allows us to evaluate the energetical efficiency and take advantage of the hydric energy availability.

This project is divided into two different parts. The first part is related to the irrigation system, the estimation of the hydric and soil requirements will be carried out in accordance with the crops needs. Filling an area of approximately 30 hectares, the irrigation system will 
supply three different types of woody crops: citrics, avocado and khaki. The design of this part will be composed of the agronomic design in accordance with the characteristics of the crops, the selection of irrigation sectors and finally, the size of the pipelines.

The second part is related to the PAT, the one that will be used to produce energy from the energy jump between the water deposit and the multioutlet hydrant. To be able to study both parts and obtain consistent results, four different scenarios of irrigation sectorization will be presented in accordance with the real operating situations.

Finally, it will be able to estimate the energy recovery from this system during a year in accordance with the water supply. It will allows us to evaluate the efficiency and selfsufficiency.

\section{1- Introducción. Objetivo del Trabajo.}

Al realizar un repaso y comparativa de las fuentes de energía de carácter renovable, una de las más importantes y empleadas en todo el mundo, desde antaño, es la energía hidráulica. Desde la segunda mitad del siglo $X X$, se han desarrollado importantes infraestructuras hidráulicas para abastecimiento y producción energética a través de turbinas de gran calibre, aferrándose como una de las primeras fuentes energéticas limpias y de bajo coste. Sin embargo, la explotación continua de este tipo de tecnología a gran a escala ha restado importancia a la posibilidad de producir energía a partir de pequeñas corrientes 0 flujos generados en sistemas de distribución a presión.

En este contexto, y relacionándolo con el ámbito de la agronomía, resultó interesante llevar a cabo un estudio técnico-analítico sobre ciertas condiciones existentes en redes de riego a presión, para las cuales se dificulta la aplicación de turbinas, pese a la posibilidad existente de recuperación energética. Ello se debe al elevado coste de esta tecnología y su amortización, para el caso de instalaciones de pequeño calibre, donde el rango de caudales y alturas recuperables no se adecua con las prestaciones generales de las turbinas hidráulicas.

Por esa razón, una de las soluciones investigadas desde hace un corto periodo de tiempo es la aplicación de bombas centrífugas de pequeño calibre, que actúen a la inversa de su funcionamiento normal, obteniendo energía de sistemas de distribución por gravedad. En estos últimos, se suelen emplear válvulas reductoras de presión para reducir la sobrepresión generada y permitir un correcto funcionamiento del sistema, por lo tanto, parte de la energía generada se pierde y la instalación pierde eficiencia.

Con el fin de ampliar la información y los estudios sobre este tipo de tecnología, nace el objetivo principal del presente trabajo : diseñar y dimensionar una red de riego a presión abastecida desde un depósito por gravedad e implantar una bomba trabajando como turbina, con el fin de cuantificar la recuperación energética posible en función de las condiciones de funcionamiento de la instalación.

Desafortunadamente, la inclusión de bombas actuando como turbinas ("Pump working As a Turbine", PAT) en conducciones a presión se ve limitada debido a la falta de información sobre los puntos de operación de la máquina hidráulica, reflejados a través de sus curvas características (Q-H; Q- $\eta$ ). Así pues, al no conocer ni poder predecir el comportamiento de la PAT, se dificulta su empleo y aplicación.

De esta forma, se propone el estudio analítico de la implantación de una PAT en una red de riego de la Comunidad de Regantes de Picassent, aprovechando el desnivel existente entre una de las balsas de regulación y la zona objeto de proyecto.

Por consiguiente, a fin de aportar rigurosidad al estudio experimental, se ha procedido a establecer diferentes escenarios en la organización del riego, los cuales permitan concluir sobre las condiciones que maximizan el aprovechamiento energético del desnivel existente. 


\section{2- Descripción del tema. Proceso de ejecución del Trabajo.}

Tras la recopilación de los parámetros referentes a los cultivos y la zona de proyecto, asi como el establecimiento de los escenarios de riego pertinentes, se realiza un dimensionado técnico-óptimo para cada escenario de la red de transporte utilizando la herramienta informática RGWin (Arviza, 2020). Asimismo, se aplica una metodología experimental para la obtención de las curvas de operación analíticas de la PAT, mediante el empleo de métodos empíricos, a partir de una bomba centrífuga seleccionada de cualquier catálogo. Tales curvas se requieren para la simulación de la instalación en cada uno de los casos, observando la energía y potencia recuperables en función de las condiciones de trabajo.

\section{1- Diseño Agronómico de la zona regable.}

Primeramente, es de vital importancia obtener los datos y parámetros permitiendo caracterizar la zona objeto de estudio. De esta forma, para abordar el diseño agronómico, se requiere extraer información acerca de la cartografía, climatología, edafología e hidrología de la zona, puesto que uno de los objetivos y alcances de este proyecto es la sostenibilidad y eficiencia en el empleo de los recursos naturales. Para ello, se ha acudido a fuentes de información como las bases cartográficas del IGN (Instituto Geográfico Nacional) y del ICV (Instituto Cartográfico Valenciano) para obtener los datos y documentos necesarios y poder visualizar la topografía del terreno, además de realizar correctamente el diseño de la red de riego.

Las parcelas de riego objeto de estudio se encuentran en la Comunidad de Regantes del término municipal de Picassent, perteneciente a la comarca de L' Horta Sud, situada al sur de la ciudad de Valencia. Para la realización del proyecto se han seleccionado un total de 44 parcelas, abarcando una superficie total de 30,48 ha.

Consultando los datos meteorológicos ofrecidos en la red SIAR (Servicio de Información Agroclimática para el Regadío), se recogen los asociados a la estación meteorológica de Picassent: evapo-transpiración (ETo), precipitación efectiva $(\mathrm{Pe})$, temperaturas medias y más extremas (Tm, Tmáx, Tmin).

En referencia a la edafología y textura del suelo, se obtiene del ICV la litología de la zona, concluyendo que la textura del suelo en la zona objeto de estudio es franca-arcillosa arenosa. Ello favorecerá la retención de agua a nivel radicular, siendo en cantidades controladas bueno para los cultivos.

A nivel hidrológico, el agua de riego empleada para abastecer a los cultivos proviene de la Balsa San Rafael (capacidad de $15.000 \mathrm{~m}^{3}$ ), uno de los cinco depósitos de acumulación del Sector IV de la Comunidad de Regantes de Picassent- Alcásser. Cabe resaltar que existe un salto aprovechable entre la balsa de alimentación y el nudo aguas abajo donde se ubica la PAT de 65 metros, lo cual se traduce en un aumento de energía de presión aprovechable y controlable.

Por último, se especifica los cultivos seleccionados y el porcentaje de ocupación, lo cual modificará las necesidades totales, y las específicas de cada cultivo. Primeramente, se resalta la dominancia del naranjo (Citrus $x$ sinensis Osb.), ocupando el $60 \%$ de la superficie regable, debido a ser uno de los cultivos más importantes y mejores adaptados de la zona. Seguido del caqui (Diospyros kaki L.), ocupando el 30\% de la superficie regable, siendo uno de los cultivos más habituales en la comarca de la Ribera del Júcar y obteniendo una notable fama debido al valor organoléptico que reciben sus frutos. Por último, el aguacatero (Persea americana Mill.), ocupando el $10 \%$ de la superficie regable, una especie en auge en los últimos años en ciertas partes del litoral mediterráneo por su valor socioeconómico.

Una vez caracterizada la zona y recogidos los valores de los parámetros necesarios, se determinan los requerimientos totales de los cultivos, así como los tiempos de riego especializando para cada cultivo. 
Los datos más relevantes hacen referencia siempre al periodo de máximas necesidades, pues es ahí donde la planta requiere mayor aporte hídrico para su crecimiento y desarrollo. De esta forma, se obtienen las necesidades totales de riego (en el presente caso para el mes de julio), el caudal unitario por unidad de superficie (específico para cada cultivo) y el tiempo de riego diario por parcela.

\section{2- Dimensionado de la red de distribución a presión.}

El dimensionado de la red de transporte se ha realizado siguiendo el criterio de optimización técnico-económica, el cual busca reducir al máximo los costes totales de la instalación (energéticos y de amortización), pese a ser los primeros nulos en nuestro caso. Al ser una conducción por gravedad, no se requiere la introducción de grupos de elevación.

Al aplicar este criterio se asegura una reducción al máximo de los costes asociados a las tuberías, induciendo positivamente en la sostenibilidad y eficiencia de la instalación. Sin embargo, la aplicación informática empleada en el dimensionado (RGWin) requiere de datos correctos y reales acerca de las longitudes de los tramos de las tuberías y las cotas de los nudos de consumo (y bifurcación). Para ello, se realiza el trazado de la red empleando el programa informático QGis 3.4, el cual permite cargar una serie de capas para simular a la perfección las condiciones del lugar donde se aborda el proyecto.

Conocido el criterio seguido en el dimensionado, es importante especificar como se aborda la sectorización y organización del riego. Primeramente, el número máximo de sectores (NSmáx) será función de la jornada efectiva de riego (JER) y el tiempo de riego diario en el periodo de máximas necesidades. Dependiendo de la jornada efectiva de riego seleccionada, la sectorización se verá modificada lo que afectará directamente tanto al dimensionado de la red de riego como a la PAT seleccionada y la energía recuperada por esta.

Por otro lado, la organización del riego en el presente proyecto se ha llevado a cabo por turnos, agrupando parcelas de riego por hidrante multiusuario en base a las restricciones asociadas a estos. Con el objetivo de abordar las posibles distribuciones por turnos de las parcelas de riego, se han establecido cuatro escenarios diferentes, atendiendo a la organización de las parcelas (automatización a nivel de hidrante o a nivel de parcela) y a la JER (variando la jornada de 8 a 16 horas para cada caso). Los escenarios establecidos quedan de la siguiente forma:

- Escenario 1: Automatización con electroválvula a nivel de hidrante, JER de 8 horas y 3 sectores.

- Escenario 2: Automatización con electroválvula a nivel de hidrante, JER de 16 horas y 5 sectores.

- Escenario 3: Automatización con electroválvula a nivel de parcela (usuario), JER de 8 horas y 3 sectores.

- Escenario 4: Automatización con electroválvula a nivel de parcela (usuario), JER de 16 horas y 5 sectores.

Los hidrantes multi-usuario llevan asociados unas condicionantes que afectan directamente a su distribución en un sistema de riego localizado, así como las posibles parcelas a las que puede abastecer. Por regla general, se estima que cada hidrante puede abastecer a un máximo de 10 parcelas, para lo que requiere un mínimo de presión de trabajo de 25 metros de columna de agua. Asegurando este valor en cada nudo de consumo (hidrante), se garantiza el correcto funcionamiento del sistema y el abastecimiento a las subunidades más desfavorables.

Por último, con el fin de simular la disposición de la PAT aguas abajo del nudo de alimentación, se estima un valor de pérdidas de carga en la línea donde se coloca la máquina. La primera estimación de pérdidas de carga asociada a la PAT para el dimensionado corresponde a $20 \mathrm{mca}$. De esta forma, el programa informático empleado en el dimensionado 
de la red de riego, puede determinar matemáticamente la presión en las conducciones aguas debajo de la PAT.

\section{3- Instalación de la PAT (Pump working as a turbine)}

Previamente se ha hecho hincapié en la dificultad de incluir turbinas convencionales en este tipo de sistemas de distribución. Es por ello, las bombas trabajando como turbinas se presentan como una alternativa en redes de pequeño calibre para la recuperación energética. Si bien se dificulta la predicción de su comportamiento, son unas máquinas rudimentarias ampliamente producidas, ante la variabilidad de caudales y presiones requeridas, lo que conlleva una reducción importante en los costes de la propia máquina (tomando una turbina convencional de dimensiones similares) y del mantenimiento.

Desafortunadamente, hoy en día pocos estudios y fabricantes de bombas muestran el comportamiento de estas máquinas en diferentes condiciones de trabajo, teniendo que acudir a ensayos experimentales que pronostiquen el funcionamiento de la bomba en modo turbina a través de la determinación de las curvas características de operación.

La principal diferencia entre los dos estados de funcionamiento de la máquina es la dirección del flujo y el sentido de rotación del eje, donde el triángulo de velocidades del fluido a la salida de los álabes se ve modificado. Pese a la ligera diferencia, se asume una equivalencia en los ángulos de los flujos entrantes y salientes al elemento impulsor. Tal equivalencia permite relacionar la máquina en los dos sentidos de operación, para lo cual se establece una altura de presión generada y obtenida similar. La ligera diferencia en la altura dependerá del rendimiento de la máquina y de la variabilidad en las condiciones de funcionamiento, referentes a la altura y el caudal circulante.

Previamente se ha comentado la dificultad que conlleva la obtención de las curvas de operación de las bombas en modo turbina, a causa de la reducida información aportada por los fabricantes, lo que se presenta como la una desventaja importante asociada al empleo de las PAT. Como resultado, varios investigadores en el campo de las máquinas hidráulicas han propuesto diversos métodos empíricos que permiten obtener las curvas características a partir del Punto Óptimo de Funcionamiento (Best Efficient Point, BEP) de la bomba. Para ello, se requiere conocer las condiciones de funcionamiento de la instalación donde se instalará la PAT, variando en el presente caso según el escenario contemplado.

Los parámetros hidráulicos requeridos para cada escenario objeto de estudio son el caudal máximo circulante y la altura recuperable esperada (inicialmente se establecen 20 mca recuperables, tal valor es el aplicado para llevar a cabo el dimensionado de la red). Además, se estima un rendimiento de la bomba actuando como turbina del $70 \%$ para iniciar el procedimiento iterativo.

Una vez aclarados estos parámetros, se procede a aplicar el método empírico descrito por Sharma Williams (1994) para la determinación de los coeficientes de caudal, altura y rendimiento, obteniéndose de esta forma los parámetros referentes a la bomba en su Punto Óptimo de Operación.

Llegados a este punto, para cada escenario se selecciona una bomba centrífuga de catálogo que se adecue a las condiciones de trabajo en su punto óptimo de funcionamiento. Así pues, se pretende determinar la velocidad específica de la bomba en su punto óptimo de funcionamiento según la expresión propuesta por Mijailov (1989), el cual relaciona el rendimiento, caudal y altura impulsada por la bomba.

A través de los ensayos experimentales llevados a cabo por los investigadores, se han estimado unas curvas en base a dos coeficientes adimensionales de altura $(\psi)$ y descarga $(\phi)$, que relacionan para diferentes valores de velocidad específica, la altura útil $(\mathrm{H}$; mca) y el caudal circulante $\left(Q ; \mathrm{m}^{3} / \mathrm{s}\right)$ en función del diámetro del rodete del elemento impulsor $(D ; \mathrm{m})$ y la velocidad de rotación de este (N; rps).

Por consiguiente, empleando el valor de la velocidad específica de la bomba para cada escenario, se selecciona la curva característica experimental con un comportamiento semejante al caso estudiado. Para diferentes puntos de la curva elegida, se obtienen los valores de los coeficientes adimensionales que permiten determinar los valores reales de 
caudal turbinado y altura recuperada caracterizando la bomba seleccionada mediante la obtención de la curva característica Q-H cuando trabaja como turbina.

De la misma forma, se determina la curva característica Q- $\eta$ relacionando el caudal circulante con el rendimiento de la PAT. En este caso, se emplea una segunda gráfica discretizada también en función de las velocidades específicas de la bomba, que determina para diferentes valores de coeficientes adimensionales, la curva del rendimiento de cada PAT.

\section{4- Análisis Energético de la zona regable}

Llegados a este punto, se emplean las curvas características obtenidas para los cuatro escenarios, con el objetivo de simular la red de riego y cuantificar la energía recuperable. En lo que respecta a las curvas características obtenidas, se ha estimado una recuperación real de $15 \mathrm{mca}$, para no sobredimensionar la instalación con una bomba de mayor calibre.

El último apartado del presente proyecto tiene por objetivo modelizar la red de riego para cada escenario contemplado, empleando el programa informático Epanet 2.0. Esto permite estudiar la presión resultante en los nudos de consumo (hidrantes multi-usuario), la cuál varía en función del caudal circulante y la pérdida de carga que genera la PAT (presión recuperada).

Así pues, se exportan los datos de cada escenario al programa Epanet 2.0, simulando la PAT como una válvula de rotura de carga. El conexionado de la máquina se realiza en serie, pero Epanet 2.0 no contempla añadir una turbina, por lo que a la válvula se le asocia la curva resistente Q-H obtenida en cada escenario.

Así, se puede ir variando en cada escenario la pérdida de carga generada por la PAT y evaluar las presiones en los nudos de consumo, hasta alcanzar el valor máximo de pérdida de carga permisible o altura recuperable. Por consiguiente, quedan determinados los nudos más desfavorables para cada escenario, dependiendo de la pérdida de carga atribuida a la PAT, hasta alcanzar un valor lo más cercano a la presión funcionamiento garantizada en los hidrantes de $25 \mathrm{mca}$. Para finalizar, una vez determinado el comportamiento real de la PAT, se modeliza la instalación según la jornada efectiva de riego que tenga cada escenario.

Tras la modelización de la red de riego en función de la pérdida de carga obtenida, se recogen los valores de pérdida unitaria o altura recuperable por la válvula de propósito general que simula la PAT, permitiendo determinar la potencia eléctrica generada y la energía recuperable de la instalación.

Conocidos los valores de altura recuperable y caudal circulante, diferenciando para cada escenario en función de la sectorización, se obtiene la potencia teórica útil generada por la PAT durante la JER. Esta potencia se ve modificada según el rendimiento del generador al que se conecta la máquina para la transmisión de la energía generada y el rendimiento de la propia máquina. De esta forma, se obtiene finalmente para cada escenario el valor de potencia eléctrica, que dependerá del rendimiento global del grupo turbina-generador.

Para concluir, el último paso recae en la obtención de la energía aprovechada o extraída por la PAT en cada condición de trabajo para un periodo de tiempo. Con la intención de aportar un valor comparable y manejable, se determina la energía generada en un año, en kWh. Por tanto, se emplea el mayor de los tiempos de riego, correspondiendo al tiempo más desfavorable, asociados a cada cultivo.

\section{3- Resultados y conclusiones.}

Previamente se ha realizado una explicación de la metodología empleada en la obtención de los parámetros necesarios para el análisis energético de la red de riego. Debido a la cantidad de parámetros empleados en el estudio y análisis técnico, se exponen varias tablas resumen que recogen los parámetros determinados a lo largo del proyecto, para cuantificar la energía real aprovechable.

Finalmente, se procede a seleccionar uno de los cuatro escenarios contemplados en el estudio de la recuperación energética. En lo que respecta al criterio seguido para la elección del escenario de estudio, se han comparado parámetros que influyen en la energía total 
recuperada en un año, así como los rendimientos de la PAT en función de los caudales circulantes.

De esta forma, comparando los cuatro escenarios, se puede concluir que el Escenario 1 de dimensionado es el óptimo para la inclusión de la PAT y la recuperación energética. En este caso, la jornada efectiva de riego se ve reducida a 8 horas y los caudales circulantes son mayores. Para la bomba seleccionada, los rendimientos de trabajo para el caudal de cada sector son elevados, rondando el $70 \%$. Además, es el escenario que ofrece una mayor recuperación energética, incrementando la sostenibilidad de la instalación.

Tabla 1: Necesidades Totales reales de cada cultivo en el mes de máximos requerimientos

\begin{tabular}{|c|c|c|c|}
\hline \multicolumn{4}{|c|}{ Necesidades Totales de Riego } \\
\hline Cultivo & \multirow{2}{*}{ Mes } & $\begin{array}{c}\text { Marco Plantación } \\
(\mathrm{m})\end{array}$ & $\begin{array}{c}\mathrm{N} T r \\
\text { (//dia y planta) }\end{array}$ \\
\hline Naranjo & \multirow{3}{*}{ Julio } & $6 \times 3$ & 58,91 \\
\cline { 1 - 1 } Caqui & $5 \times 3$ & 42,91 \\
\hline Aguacate & & $6 \times 6$ & 104,83 \\
\hline
\end{tabular}

Tabla 2: Parámetros de riego que caracterizan el sistema de riego en parcela

\begin{tabular}{|c|c|c|c|c|c|}
\hline \multicolumn{6}{|c|}{ Parámetros de Riego } \\
\hline Cultivo & $\begin{array}{c}\text { Q emisor } \\
(/ / \mathrm{h})\end{array}$ & $\begin{array}{c}\mathrm{N}^{\circ} \text { emisores } \\
\text { planta }\end{array}$ & $\begin{array}{c}\text { Separación } \\
\text { entre emisores } \\
(\mathrm{m})\end{array}$ & $\begin{array}{c}\mathrm{Q} \text { unitario } \\
\left(/ / \mathrm{h} \mathrm{m}^{2}\right)\end{array}$ & $\begin{array}{c}\text { Q planta } \\
(/ / \mathrm{h})\end{array}$ \\
\hline Naranjo & 4 & 6 & 1 & 1,33 & 24 \\
\hline Caqui & 3,5 & 6 & 1 & 1,4 & 21 \\
\hline Aguacate & 3,5 & 12 & 1 & 1,17 & 42 \\
\hline
\end{tabular}

Tabla 3: Tiempos de riego diario para cada cultivo en el mes de máximos requerimientos

\begin{tabular}{|c|c|c|c|c|c|}
\hline \multicolumn{6}{|c|}{ Tiempo de Riego Diario } \\
\hline Cultivo & Mes & $\begin{array}{c}\mathrm{NTr} \\
\text { (I/día y planta) }\end{array}$ & $\begin{array}{l}\mathrm{N}^{\circ} \text { Riego } \\
\text { semanales }\end{array}$ & $\begin{array}{c}\text { Intervalo } \\
\text { entre riegos }\end{array}$ & $\begin{array}{l}\text { Tiempo de } \\
\text { riego }(\mathrm{h})\end{array}$ \\
\hline Naranjo & \multirow{3}{*}{ Julio } & 58,91 & 7 & 1 & 2,45 \\
\hline Caqui & & 42,91 & 6 & 1,17 & 2,38 \\
\hline Aguacate & & 104,83 & 7 & 1 & 2,5 \\
\hline
\end{tabular}

Tabla 4: Potencia aportada y Energía obtenida por la PAT para cada escenario

\begin{tabular}{|c|c|c|c|c|c|c|}
\hline \multicolumn{7}{|c|}{ Energía total recuperable para cada escenario } \\
\hline Escenario & $\begin{array}{c}\text { Automatización } \\
\text { Electroválvula }\end{array}$ & JER & $\begin{array}{c}\text { Número } \\
\text { Sectores } \\
\text { NS }\end{array}$ & $\begin{array}{c}\text { Horas } \\
\text { Riego Año } \\
(\mathrm{h})\end{array}$ & $\begin{array}{c}\text { Promedio } \\
\text { Potencias } \\
\text { Eléctricas }(\mathrm{W})\end{array}$ & $\begin{array}{c}\text { Energía Anual } \\
\text { Recuperada } \\
(\mathrm{kWh})\end{array}$ \\
\hline 1 & Hidrante & 8 & 3 & 915,9 & 3303,23 & 3025,42 \\
\hline 2 & Hidrante & 16 & 5 & 1526,51 & 1738,67 & 2654,1 \\
\hline 3 & Parcela & 8 & 3 & 915,9 & 2941,58 & 2694,19 \\
\hline 4 & Parcela & 16 & 5 & 1526,51 & 869,31 & 1326,65 \\
\hline
\end{tabular}


Llevada a cabo la selección del escenario óptimo para la inclusión de la PAT en función de las condiciones de trabajo, se procede a determinar el ahorro en la huella de carbono para el escenario 1.

La huella de carbono es un indicador ambiental que permite reflejar la totalidad de gases de efecto invernadero generados por una organización, un individuo o un proceso. Con el objetivo de categorizar las actuaciones y actividades de personas y empresas, se puede concluir con la sostenibilidad del caso objeto de estudio. La huella de carbono se mide en $\mathrm{kg}$ de $\mathrm{CO}_{2}$ equivalente, lo que permite contemplar el abanico de gases de efecto invernadero.

Para llevar a cabo el cálculo del ahorro de emisiones, se emplea el factor de emisión más actualizado ofrecido por la DEFRA (Department for Environment, Food \& Rural Affairs), el cual establece que la producción de $1 \mathrm{kWh}$ genera una emisión de 0,28 kg.eq. de $\mathrm{CO}_{2}$.

Con el objetivo de sostenibilizar la instalación y canalizar la energía generada (que por regla general se suele disipar mediante válvulas reductoras de presión) para su posterior empleo, se aplica el factor de emisión determinando los kilogramos equivalentes de $\mathrm{CO}_{2}$ que se dejan de emitir a la atmósfera.

Para el caso del escenario 1, siendo elegido el escenario óptimo de funcionamiento se calcula en función de los $\mathrm{kWh}$ generados en un año, los kg. eq. de $\mathrm{CO}_{2}$ evitados, siendo:

$0.28 \mathrm{~kg} \mathrm{CO} / 1 \mathrm{kWh} \times 3025.42 \mathrm{kWh}=847.12 \mathrm{~kg} \mathrm{CO} 2$ equivalentes anuales

A continuación, se adjuntan las curvas característicias obtenidas de la PAT, para el caso del escenario 1 , relacionando el caudal turbinado con la altura recuperable y con el rendimiento de la turbina tras la modelización con el programa EPANET 2.0.

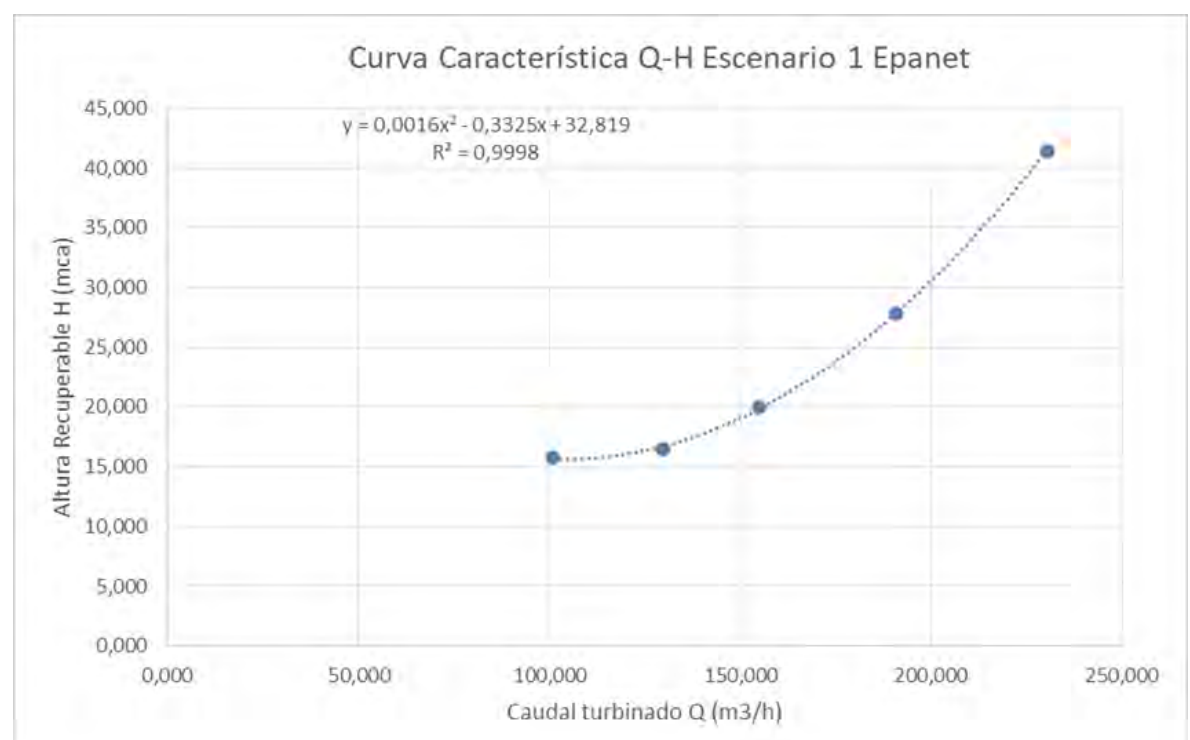

Figura 1: Curva Característica Q-H Escenario 1, modelización Epanet 2.0. 


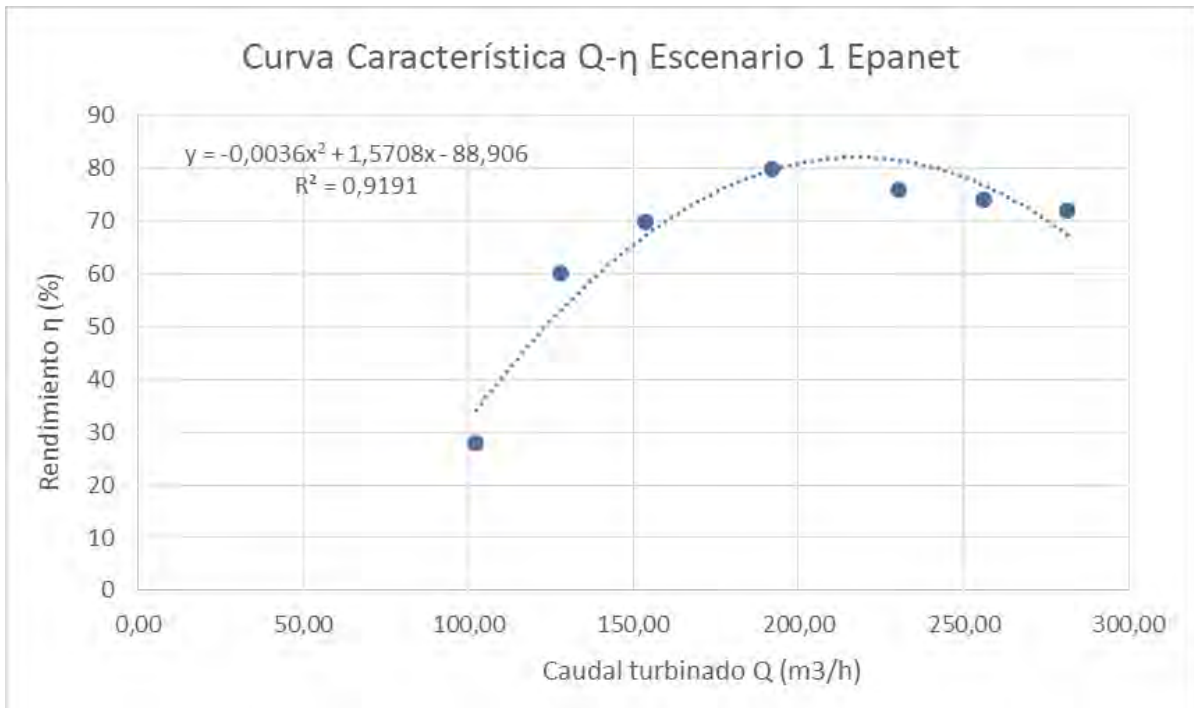

Figura 2: Curva Característica Q-n Escenario 1, modelización Epanet 2.0

Llegados a este punto, resulta importante resaltar el alcance de los objetivos y la satisfacción en los resultados obtenidos al aplicar la metodología analítica, lo cual permite concluir sobre la viabilidad de este estudio y de la propia tecnología.

En consecuencia, no en todos los casos resulta interesante el empleo de la PAT, ya que al tener un rendimiento bajo en algunas situaciones de trabajo la eficiencia se ve reducida. De esta forma, los factores que deben controlarse y estudiar para valorar la introducción de esta tecnología son: el caudal circulante, el tiempo de riego y el tipo de instalación donde se proyecta.

Como se ha comprobado analizando los resultados de los escenarios 2 y 4 , pese a tener un tiempo de riego mayor, a caudales circulantes bajos y variables en el tiempo de riego, el rendimiento de la PAT se ve muy reducido, condicionando el aprovechamiento energético.

Por lo tanto, las condiciones óptimas de funcionamiento para la instalación de una PAT son: caudales altos y poco variables en el tiempo, además de un altura util aprovechable, siendo la jornada efectiva de riego poco relevante cuando la potencia instalada es baja. Estas condiciones quedan reflejadas en el Escenario 1, siendo el seleccionado como el escenario óptimo entre los contemplados por la mayor recuperación energética.

A nivel medioambiental, este proyecto puede enfocarse de dos modos o vertientes. Por un lado, al realizar un estudio característico y específico para cada cultivo y sus condiciones de crecimiento, se determinan con exactitud los requerimientos hídricos anuales. Ello permite adecuar y reducir el volumen de agua de riego, lo que tiene una repercusión directa en el mantenimiento y preservación de los recursos hídricos. Este tema resulta de vital importancia debido a la progresiva disminución de la capa freática y el nivel de los acuíferos. Por otro lado, el hecho de reducir las pérdidas energéticas y aprovechar parte de éstas, se traduce en una disminución del consumo energético en los casos que se pueda emplear en la misma instalación (automatización, alumbrado, ...). Aunque no se requiera consumo energético, puede verterse a red para abastecimiento de cabezales o pequeñas instalaciones cercanas.

Con la mira puesta en el futuro y en aplicaciones a mayor escala, las PAT se muestran como una solución en redes de riego que abastecen a gran superficie regable, dónde en ciertas zonas sea posible su inclusión reduciendo los requerimientos energéticos de la instalación. Con el paso del tiempo, se desarrollarán más estudios que muestren la eficiencia y beneficio del empleo de esta tecnología, motivando a los fabricantes a estudiar el empleo de este tipo de bombas en modo turbina y aportar la información necesaria relativa a su comportamiento, permitiendo seleccionar a cada proyectista la que mejor se ajuste a las condiciones de trabajo. 


\section{4- Bibliografía}

AGUSTÍ, M. (2003). Manual de fruticultura. Editorial Mundi-Prensa.

ARVIZA, J. (2020). RGWin programa informático para el dimensionado de redes.

BOMBAS IDEAL (2017). Catálogo comercial

CARRAVETTA, A.; DERAKHSHAN, S.; M. RAMOS, H. (2018). Pumps as turbines: Fundamentals \& Applications. Editorial Springer.

EPANET 2.0.

MEIRELLES, G.; LUZIVOTTO, E.; M. BRENTAN, B. (2017). Selection \& location of pumps as turbines substituting pressure reducing valves. Renewable Energy 109: 392-405

MIJAILOV, L.P. (1989). Pequeña Hidroenergía (en ruso). Moscú: Ed Energoatomizdat

ORTIZ, R.; JIMÉNEZ, A. (2008). Máquinas hidráulicas reversibles aplicadas a micro centrales hidroeléctricas. IEEE Latin America Transaction 6: 170-175

POLESE, J.-M. (2007). Cultivo de cítricos. Editorial Omega.

PUGLIESE, F.; DE PAOLA, F.; FONTANA, N.; GIUGNI, M.; MARINI, G. (2016). Experimental characterization of two pumps as turbines for hydropower generation. Renewable Energy 99: 180-187

RAGAZZINI, D. (1985). El Kaki. Editorial Mundi-Prensa.

ROMERO-MARRERO, L.; PÉREZ-SÁNCHEZ, M.; LÓPEZ-JIMÉNEZ, P.A. (2018). Estimación de las curvas características de operación de sistemas de impulsión operando como turbinas a partir de su curva motriz trabajando como bomba. Ingeniería del agua 22: 15-26

TÉLIZ, D; MORA, A. (2017). El aguacate y su manejo integrado. Editorial Biblioteca básica de agricultura

TRICARICO, C.; GARGANO, R.; KAPELAN, Z.; SANTOPIETRO, S.; GRANATA, F.; DE MARINIS, G. (2017). Optimal energy recovery by means of pump as turbines (PATs) for improved WDS management. Water Science \& Technology: water supply

WILLIAMS, A. A. (1994). The turbine performance of centrifugal pumps: a comparison of prediction methods. Proceedings of the Institution of Mechanical Engineers, Part A 208: $59-66$

DOI: $10.31428 / 10317 / 8712$ 